\section{The Om of fly smooshing}

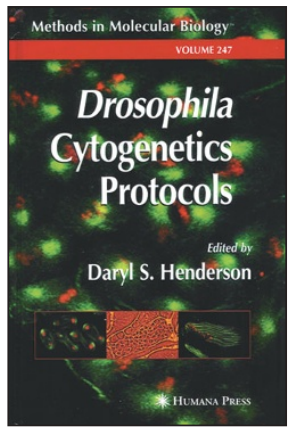

\section{Drosophila Cytogenetics Protocols}

Daryl S Henderson (ed.)

Humana Press, 2003

248 pp. hardcover, $\$ 125.00$

ISBN 1-58829-050-6

\section{Reviewed by Chris Bazinet}

In a day when so much technical information comes from slick manuals churned out so fast that you can't keep track of the updates, or over the phone from 'technical reps' at the company that sold you a kit that was "a sure thing", Drosophila Cytogenetics Protocols, edited by Daryl Henderson, presents a refreshingly solid, diverse and sometimes even entertaining spectrum of cytogenetic approaches to fruit fly biology by skilled and highly experienced fly researchers. The stylishly retro cloth binding communicates the subtle promise that this volume will be worth returning to for years to come. I'm inclined to believe it will live up to that promise. It invites a true reading in a comfortable chair, in contrast to the expensive workbook-style bench guide that seems intended to be read standing up with a pipettor in one hand and a cell phone in the other. Face it: good cytology requires patience and a certain attitude. Don't fall for those 'polyester' lab manuals when you could be luxuriating in the "...clean, old, wellwashed, long-tailed cotton shirt..." version offered here (to borrow an expression from Adelaide Carpenter). Indeed, the spirit of this volume is not that it will get you through, but that it will make you comfortable with the technology and put you in touch with the art form.

The long history of conceptual and technical innovation in Drosophila cytogenetics really comes through when you browse this book, a handy but comprehensive fly cytogenetics course in a compact package, perhaps the best single volume on fruit fly cytology to date. The contrasting technical approaches presented for observing the status and behavior of chromosomes in a number of different cell types offers a rich resource for researchers looking to master existing cytological technique, synthesize new cytological approaches based on combinations of existing methods or understand at a deeper level the biochemical or 'black magic' basis for what we know about fly chromosomes.

Detailed protocols for standard cytological procedures likely to be used in most fly research labs (acetic-orcein staining of polytene

Chris Bazinet is in the Department of Biological Sciences at St. John's University, 8000 Utopia Pkwy, Jamaica, New York 11439, USA. e-mail:bazinetc@stjohns.edu chromosomes, analysis of aberrations in salivary gland polytenes, in situ hybridization to polytene chromosomes, mitotic chromosome preparations from larval brains) are presented with a helpful perspective on the original work extending back to Calvin Bridges and, in general, a keen feeling for the organism. A natural extension of cytogenetics is the developmental analysis of germ cells. An excellent chapter on spermatogenesis offers a nice overview of this complex morphogenetic pathway, illustrating a number of mutant phenotypes throughout the pathway, and good, detailed, current protocols for examining all stages. Ovaries don't get short shrift, nor do larval brains or imaginal discs, with chapters on immunostaining of each. Several chapters present cutting-edge techniques that will probably become increasingly mainstream in the future, like the combined immunostaining and FISH analysis of polytene chromosomes, whole-mount FISH analysis in embryos, RNA interference and the use of visual markers for apoptosis.

Interspersed are a few chapters on methods that strike me as truly specialist protocols, used by only a few labs, but which nevertheless fill in many of the common gaps that account for the "How do we know that?" moments that often present themselves in the lab or, more importantly, which might be combined with other methods to spur a new wave of cytological approaches to the fly. Chapters on FeulgenDNA cytophotometry, electron microscopy of polytene chromosomes (is that how they do that?!), formation and analysis of dicentric chromosomes using inverted FRTs, cytology of histoblasts and SMART, a fly-based assay for mutagenicity and induction of recombination, are good examples. The chapter by Koryakov, Mal'ceva, King and Zhimulev on polytene chromosomes from ovarian nurse cells reminds us that there is much to learn about chromosome organization in differentiated cells. This interleaving of highly specialist procedures with the (now) more mainstream antibody- and hybridization-based protocols is also instructive in providing a wonderful overview of great variations in specimen preparation and fixation, the often underappreciated and poorly understood aspects of cytology that make it the subtle art that it is.

As is often the case in a volume of this sort, I found a few of the presentations on the perfunctory side. And for a specialist's cytology book, the limited use of color is disappointing. Also, the few color plates (seven in all, spread over four pages) are presented with partial captions, just enough to confirm that you're referring to the correct full caption that accompanies the black-and-white version in the appropriate chapter (which might be hundreds of pages away). To get through the 16 panels in Color Plate 3, referring to the figure legend some 150 pages removed from it, is a bit taxing, but you're reading this book in a comfortable chair, right? On balance, this is a relatively minor, probably cost-driven defect, more than made up for by the many high quality efforts of the authors. The combination of these diverse and carefully articulated presentations makes this a valuable and stimulating source of essential technical information for all experimental 'drosophilists'. 